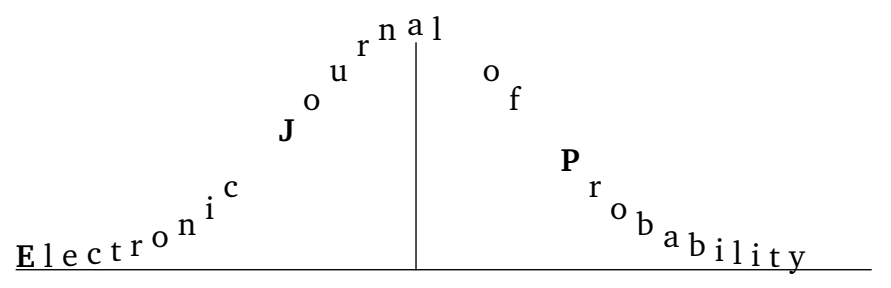

Vol. 14 (2009), Paper no. 76, pages 2241-2263.

Journal URL

http://www.math.washington.edu/ ejpecp/

\title{
On The Traces Of Laguerre Processes
}

\section{Víctor Perez-Abreu}

Centro de Investigación en Matematicas A.C., Department of Probability and Statistics, Apdo Postal 402, Guanajuato 36000 Gto. Mexico

Email: pabreu@cimat.mx

\section{Constantin Tudor}

University of Bucharest,

Faculty of Mathematics and Computer Science, Str. Academiei 14, 010014 Bucharest, Romania Email: ctudor@fmi.unibuc.ro

\begin{abstract}
Almost sure and $L^{k}$-convergence of the traces of Laguerre processes to the family of dilations of the standard free Poisson distribution are established. We also prove that the fluctuations around the limiting process, converge weakly to a continuous centered Gaussian process. The almost sure convergence on compact time intervals of the largest and smallest eigenvalues processes is also established.
\end{abstract}

Key words: Matrix valued process, Complex Wishart distribution, Trace processes, Largest and smallest eigenvalues, Propagation of chaos, Fluctuations of moments, Free Poisson distribution.

AMS 2000 Subject Classification: Primary 60K35, 60F05.

Submitted to EJP on August 21, 2008, final version accepted September 17, 2009. 


\section{Introduction}

For $m, n \geq 1$, let $\left\{B_{m, n}(t)\right\}_{t \geq 0}=\left\{\left(B_{m, n}^{j, k}(t)\right)_{1 \leq j \leq m, 1 \leq k \leq n}\right\}_{t \geq 0}$ be an $m \times n$ complex Brownian motion; that is, the entries $\left\{\operatorname{Re}\left(B_{m, n}^{j, k}(t)\right)\right\}_{t \geq 0},\left\{\operatorname{Im}\left(B_{m, n}^{j, k}(t)\right)\right\}_{t \geq 0}$ are independent one-dimensional Brownian motions.

The continuous $n \times n$-matrix-valued process $L_{m, n}(t)=B_{m, n}^{*}(t) B_{m, n}(t), t \geq 0$, is known as Laguerre process (or complex Wishart process) of size $n$, of dimension $m$ and starting from $L_{m, n}(0)=$ $B_{m, n}^{*}(0) B_{m, n}(0)$. For $n=1, L_{m, 1}$ is a squared Bessel process.

Let $\left\{\Sigma_{m, n}(t)\right\}_{t \geq 0}=\left\{\frac{1}{2 n} L_{m, n}(t)\right\}_{t \geq 0}$ be a Laguerre processes scaled by $\frac{1}{2 n}$ and let

$$
\left\{\lambda^{(m, n)}(t)\right\}_{t \geq 0}=\left\{\left(\lambda_{1}^{(m, n)}(t), \lambda_{2}^{(m, n)}(t), \ldots, \lambda_{n}^{(m, n)}(t)\right)\right\}_{t \geq 0}
$$

be the $n$-dimensional stochastic process of eigenvalues of $\left\{\Sigma_{m, n}(t)\right\}_{t \geq 0}$.

In the case of real Wishart processes (i.e. $B_{m, n}(t)$ has real entries) and $m>n-1$, Bru [4] proved that if the eigenvalues start at different positions

$$
0 \leq \lambda_{1}^{(m, n)}(0)<\lambda_{2}^{(m, n)}(0)<\ldots<\lambda_{n}^{(m, n)}(0)
$$

then they never meet at any time

$$
0 \leq \lambda_{1}^{(m, n)}(t)<\lambda_{2}^{(m, n)}(t)<\ldots<\lambda_{n}^{(m, n)}(t) \text { a.s. } \forall t>0,
$$

and furthermore they are governed by a diffusion process satisfying an Itô Stochastic Differential Equation (ISDE).

The same happens in the case of Laguerre processes (with the same arguments as in the real case) and the ISDE's has the form

$$
\mathrm{d} \lambda_{j}^{(m, n)}(t)=\sqrt{\frac{2 \lambda_{j}^{(m, n)}(t)}{n}} \mathrm{~d} W_{j}^{(m, n)}(t)+\frac{1}{n}\left(m+\sum_{k \neq j} \frac{\lambda_{j}^{(m, n)}(t)+\lambda_{k}^{(m, n)}(t)}{\lambda_{j}^{(m, n)}(t)-\lambda_{k}^{(m, n)}(t)}\right) \mathrm{d} t,
$$

$t \geq 0,1 \leq j \leq n$, where $W_{1}^{(m, n)}, \ldots, W_{n}^{(m, n)}$ are independent one-dimensional standard Brownian motions; see for example [7], [8], [9], [19], [20], [21].

A special feature of this system of ISDEs is that it has non smooth drift coefficients and the eigenvalues processes do not collide. When $m>n-1$, this SDE has a unique solution in the sense of probability law [7, Theorem 4].

Throughout the paper we shall assume that $m>n-1$ and (1). We denote by $\mathrm{Tr}$ the usual unnormalized trace of a matrix and $\operatorname{tr}=\frac{1}{n} \operatorname{Tr}$ is the normalized one.

Consider the empirical process

$$
\mu_{t}^{(m, n)}=\frac{1}{n} \sum_{j=1}^{n} \delta_{\lambda_{j}^{(m, n)}(t)}, t \geq 0
$$


where $\delta_{x}$ is the unit mass at $x$. In the present paper we are concerned with functional limit theorems for the $p$-moment or $p$-trace processes associated with $\mu_{t}^{(m, n)}$ for any $p \geq 1$. The dynamical behavior of the extreme eigenvalues processes is also investigated.

Specifically, we consider propagation of chaos and asymptotic fluctuations for the one-dimensional processes $\left(\left\{M_{m, n, p}(t)\right\}_{t \geq 0}: n \geq 1\right)$ defined by the semimartingales

$$
M_{m, n, p}(t)=\operatorname{tr}\left(\left[\Sigma_{m, n}(t)\right]^{p}\right)=\int_{\mathbb{R}} x^{p} \mu_{t}^{(m, n)}(\mathrm{d} x)=\frac{1}{n} \sum_{j=1}^{n}\left[\lambda_{j}^{(m, n)}(t)\right]^{p} .
$$

An important role in this paper is played by the family of dilations of free Poisson law. Recall that the free Poisson distribution (or Marchenko-Pastur distribution) $\mu_{c}^{f p}, c>0$, is the probability measure on $\mathbb{R}_{+}$defined by

$$
\mu_{c}^{f p}(\mathrm{~d} x)=\left\{\begin{array}{cc}
v_{c}(\mathrm{~d} x), & c \geq 1 \\
(1-c) \delta_{0}(\mathrm{~d} x)+v_{c}(\mathrm{~d} x), & c<1
\end{array}\right.
$$

where

$$
\begin{gathered}
v_{c}(\mathrm{~d} x)=\frac{\sqrt{(x-a)(b-x)}}{2 \pi x} 1_{(a, b)}(x) \mathrm{d} x, \\
a=(1-\sqrt{c})^{2}, b=(1+\sqrt{c})^{2} .
\end{gathered}
$$

It was shown by Marchenko and Pastur [23] that $\mu_{c}^{f p}$ is the asymptotic distribution, when $t=1$, of the empirical spectral measure (3) when $\lim _{n \rightarrow \infty} \frac{m}{n}=c$. This explains why when $c<1$ this distribution has an atom at zero with mass $(1-c)$, since in this case the Laguerre matrices are singular having zero eigenvalues. In this work we always consider the case $c \geq 1$.

In this work we also consider the family $\left(\mu_{c}(t)\right)_{t>0}$ of dilations of the free Poisson distribution given by $\mu_{c}(t)=\mu_{c}^{f p} \circ h_{t}^{-1}$, where $h_{t}(x)=t x$. That is,

$$
\begin{gathered}
\mu_{c}(t)(\mathrm{d} x)=\left\{\begin{array}{cc}
v_{c}(t)(\mathrm{d} x), & c \geq 1 \\
(1-c) \delta_{0}(\mathrm{~d} x)+v_{c}(t)(\mathrm{d} x), & c<1
\end{array},\right. \\
v_{c}(t)(\mathrm{d} x)=\frac{\sqrt{(x-a t)(b t-x)}}{2 \pi t x} 1_{(a t, b t)}(x) \mathrm{d} x,
\end{gathered}
$$

with $\mu_{c}(0)=\delta_{0}$. For the $p$-moment we use the notation

$$
\mu_{c, p}(t)=\int_{0}^{\infty} x^{p} \mu_{c}(t)(\mathrm{d} x)
$$

For a fixed $t>0$, the study of different aspects of traces or moments of Wishart random matrices has been considered by Marchenko and Pastur [23], Oravecz and Petz [24], Voiculescu-Dykema and Nica [27], amongst others. An important role in those papers is played by the moments of the free Poisson law $\mu_{c}^{f p}$.

In the case of complex Hermitian Brownian motions $\left\{B_{n, n}(t) ; t \geq 0\right\}, n \geq 1$, the corresponding systems of eigenvalues are called Dyson Brownian motions. In the study of functional limit theorems for these processes (see for example [5], [25]), a key role is played by the family of semicircle laws 
which agrees with the dilations of the standard semicircle law, which is the spectral distribution of the free Brownian motion. However, in the case of Laguerre process, we show that the limit is the family of dilations of free Poisson laws which is different from the family of free Poisson laws, obtained by taking $c t$ as the parameter.

The paper is organized as follows. For the sake of completeness, in Section 2 we recall a known result on the characterization of the families of dilations of free Poisson laws in terms of an initial valued problem for their Cauchy transforms. We also present a functional recursive equation for the families of moments of free Poisson laws.

In Section 3 we prove, for the Laguerre model, uniform almost surely and in $L^{k}$ laws of large numbers. We show that the sequence of measure-valued processes $\mu_{t}^{(m, n)}$ converges almost surely, as $\lim _{n \rightarrow \infty} \frac{m}{n}=c$, to the family $\left\{\mu_{c}(t)\right\}_{t \geq 0}$ (which for $t=1$ agrees with $\mu_{c}^{f p}$ ) in the space of continuous functions from $\mathbb{R}_{+}$into probability measures in $\mathbb{R}$, endowed with the uniform convergence on compact intervals of $\mathbb{R}$ (Theorems 3.1 and 3.3). We also address the question of the weak convergence of the fluctuations of the moment processes $V_{m, n, r}(t)=\int x^{r} Y_{t}^{(m, n)}(\mathrm{d} x)$, where $Y_{t}^{(m, n)}=n\left(\mu_{t}^{(m, n)}-\mu_{c}(t)\right)$. It is shown that for each $r \geq 1, V_{m, n, r}$ converges, as $\lim _{n \rightarrow \infty} \frac{m}{n}=c$, to a one-dimensional Gaussian process $Z_{r}$ given in terms of the previous $(r-1)$ th limiting processes $Z_{1}, \ldots, Z_{r-1}$, and a Gaussian martingale (Theorem 3.7). By using an upper large deviations bound for the empirical measure valued process obtained in [5], we derive an upper estimate of the deviation of the moment processes from a given deterministic measure valued process $v$ in terms of the entropy of $v$ (Proposition 3.5).

In Section 4 we prove the almost sure convergence, as $\lim _{n \rightarrow \infty} \frac{m}{n}=c$, of the supremum over the interval $[0, T]$ of the largest eigenvalue process $\left\{\lambda_{n}^{(m, n)}(t)\right\}_{t \geq 0}$ to $(1+\sqrt{c})^{2} T$ as well as the convergence for the infimum over the interval $[0, T]$ of the smallest eigenvalue process $\left\{\lambda_{1}^{(m, n)}(t)\right\}_{t \geq 0}$ to $(1-\sqrt{c})^{2} T$ (Theorem 4.1).

\section{Preliminaries}

Recall that for a finite non-negative measure $v$ on $\mathbb{R}$, its Cauchy transform is defined by

$$
G^{v}(z)=\int_{\mathbb{R}} \frac{v(\mathrm{~d} x)}{x-z}
$$

for all non-real $z$ with $\operatorname{Im}(z) \neq 0$. It is well known that $G^{v}$ is analytic in $\mathbb{C} \backslash \mathbb{R}, \overline{G^{v}(z)}=G^{v}(\bar{z})$, $G^{v}: \mathbb{C}^{+} \rightarrow \mathbb{C}^{+}$, where $\mathbb{C}^{+}:=\{z: \operatorname{Im}(z)>0\}$ and $\lim _{\eta \rightarrow \infty} \eta\left|G^{v}(i \eta)\right|<\infty$ (see for example [17]).

The Cauchy transform for the free Poisson distribution is

$$
G^{f p}(z)=\frac{-(z+1-c)+\sqrt{(z+1-c)^{2}-4 c}}{2 z} .
$$

For the laws $\left\{\mu_{c}(t)\right\}_{t \geq 0}$, writing $G_{c, t}(z)$ for $G^{\mu_{c}(t)}(z)$, we have the relation

$$
G_{c, t}(z)=\frac{-(z+t(1-c))+\sqrt{(z+t(1-c))^{2}-4 c t^{2}}}{2 t z}, t>0, \operatorname{Im}(z) \neq 0 .
$$


The following characterization of the family of distributions $\left\{\mu_{c}(t)\right\}_{t \geq 0}$ of dilations of free Poisson distribution in terms of an initial valued problem for the corresponding Cauchy transforms was proved in [5, Corollary 3.1].

Proposition 2.1 The family $\left\{\mu_{c}(t)\right\}_{t \geq 0}$ is characterized by the property that its Cauchy transforms is the unique solution of the initial value problem

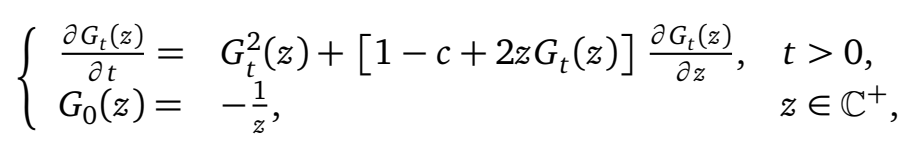

which satisfies $G_{t}(z) \in \mathbb{C}^{+}$for $z \in \mathbb{C}^{+}$and

$$
\lim _{\eta \rightarrow \infty} \eta\left|G_{t}(i \eta)\right|<\infty, \text { for each } t>0
$$

Remark 2.2. a) From Lemma 3.3.9 in [17], a characterization of the family $\left\{\mu_{c}^{f p}(t)\right\}_{t \geq 0}$ of free Poisson laws can be obtained in terms of an initial valued problem for the corresponding Cauchy transforms.

b) From [11, Remark 6.9] or [24, Proposition 11] we have the relation

$$
\mu_{c, r}(1)=\frac{1}{r} \sum_{k=1}^{r} C_{r}^{k} C_{r}^{k-1} c^{k}, r \geq 1
$$

and hence

$$
\mu_{c, r}(t)=\mu_{c, r}(1) t^{r}
$$

The following functional recursive equation for the families of moments of free Poisson laws holds.

Proposition 2.3. For each $r \geq 1$ and $t>0$

$$
\mu_{c, r}(t)=r c \int_{0}^{t} \mu_{c, r-1}(s) \mathrm{d} s+r \sum_{j=0}^{r-2} \int_{0}^{t} \mu_{c, r-1-j}(s) \mu_{c, j}(s) \mathrm{d} s .
$$

Proof. The following formula for the moments of free Poisson distribution

$$
\mu_{c, r}(1)=c \mu_{c, r-1}(1)+\sum_{j=0}^{r-2} \mu_{c, r-1-j}(1) \mu_{c, j}(1),
$$

follows from the series expansion

$$
G_{c, 1}(z)=\sum_{r=0}^{\infty} \mu_{c, r}^{f p}(1) z^{-r-1}
$$

identifying the coefficients in the relation

$$
-z G_{c, 1}(z)=-c G_{c, 1}(z)+z\left[G_{c, 1}(z)\right]^{2}+G_{c, 1}(z)+1 .
$$

Now (14) is a consequence of (13) and (15). 


\section{Functional limit theorems for the trace processes}

In this section we show the uniform a.s. and $L^{k}$ laws of large numbers for the moment processes $\left\{M_{m, n, r}(t)\right\}_{t \geq 0}=\left\{\operatorname{tr}\left(\left[\sum_{m, n}(t)\right]^{r}\right)\right\}_{t \geq 0}$ given by (4) and we prove the weak convergence of $\left\{\mu_{t}^{(m, n)}\right\}_{t \geq 0}$ to a measure valued process satisfying an evolution equation.

Let $\operatorname{Pr}(\mathbb{R})$ be the space of probability measures on $\mathbb{R}$ endowed with the topology of weak convergence and let $C\left(\mathbb{R}_{+}, \operatorname{Pr}(\mathbb{R})\right)$ be the space of continuous functions from $\mathbb{R}_{+}$into $\operatorname{Pr}(\mathbb{R})$, endowed with the topology of uniform convergence on compact intervals of $\mathbb{R}_{+}$. As it is usual, for a probability measure $\mu$ and a $\mu$-integrable function $f$ we use the notation $\langle\mu, f\rangle=\int f(x) \mu(\mathrm{d} x)$.

\subsection{Propagation of chaos}

The next goal is to prove uniform a.s. and $L^{k}$, for each $k \geq 1$, laws of large numbers for the trace processes $M_{m, n, r}$. The first part of the next result gives useful recursive equations systems for the processes $M_{m, n, r}$ and for product of powers of them in terms of the martingales

$$
X_{m, n, r-\frac{1}{2}}(t)=\frac{1}{n^{\frac{3}{2}}} \sum_{j=1}^{n} \int_{0}^{t}\left[\lambda_{j}^{(m, n)}(s)\right]^{r-\frac{1}{2}} \mathrm{~d} W_{j}^{(m, n)}(s), t \geq 0,
$$

whose increasing processes are given by

$$
\left\langle X_{m, n, r-\frac{1}{2}}\right\rangle_{t}=\frac{1}{n^{2}} \int_{0}^{t} M_{m, n, 2 r-1}(s) \mathrm{d} s, t \geq 0,
$$

for any $r \geq 0$.

In the following $(m(n))_{n}$ is a sequence of positive integers with $m(n)>n-1$ and $\lim _{n \rightarrow \infty} \frac{m(n)}{n}=c>$ 0 .

For each $k \geq 0$ and a multi-index $\underline{i}_{k+1}=\left(i_{1}, \ldots, i_{k+1}\right) \in Z_{+}^{k+1}$ such that $\sum_{r=1}^{k+1} r i_{r}=k+1$, we define

$$
r_{\underline{i}_{k+1}}^{m, n}(t)=M_{m, n, 1}^{i_{1}}(t) \ldots . M_{m, n, k+1}^{i_{k+1}}(t) .
$$

Theorem 3.1. (i) The following two relations hold for $m \geq n \geq 1, r \geq 1, k \geq 0$ and $t \geq 0$

a)

$$
\begin{gathered}
M_{m, n, r}(t)=M_{m, n, r}(0)+r X_{m, n, r-\frac{1}{2}}(t)+\frac{m r}{n} \int_{0}^{t} M_{m, n, r-1}(s) \mathrm{d} s \\
+\frac{r(r-1)}{n} \int_{0}^{t} M_{m, n, r-1}(s) \mathrm{d} s+r \sum_{j=0}^{r-2} \int_{0}^{t} M_{m, n, r-1-j}(s) M_{m, n, j}(s) \mathrm{d} s, t \geq 0,
\end{gathered}
$$


b)

$$
\begin{aligned}
& r_{\underline{i}_{k+1}}^{m, n}(t)=r_{\underline{i}_{k+1}}^{m, n}(0)+\sum_{r=1}^{k+1} r i_{r} \int_{0}^{t} r_{\underline{i}_{k+1}-e_{r}}^{m, n}(s) \mathrm{d} X_{m, n, r-\frac{1}{2}}(s) \\
& +\frac{m}{n} \sum_{r=1}^{k+1} r i_{r} \int_{0}^{t} r_{\underline{i}_{k+1}-e_{r}+e_{r-1}}^{m, n}(s) \mathrm{d} s \\
& +\frac{1}{n} \sum_{r=2}^{k+1} r(r-1) i_{r} \int_{0}^{t} r_{\underline{i}_{k+1}}^{m, n}-e_{r}+e_{r-1}(s) \mathrm{d} s \\
& +\sum_{r=2}^{k+1} \sum_{l=0}^{r-2} r i_{r} \int_{0}^{t} r_{\underline{i}_{k+1}-e_{r}+e_{r-1-l}+e_{l}}^{m, n}(s) \mathrm{d} s \\
& +\frac{1}{n^{2}} \sum_{1 \leq l<r}^{k+1} \operatorname{lr} i_{l} i_{r} \int_{0}^{t} r_{\underline{i}_{k+1}-e_{l}-e_{r}+e_{l+r-1}}^{m, n}(s) \mathrm{d} s \\
& +\frac{1}{2 n^{2}} \sum_{r=1}^{k+1} r^{2} i_{r}\left(i_{r}-1\right) \int_{0}^{t} r_{\underline{i}_{k+1}}^{m, n}-2 e_{r}++e_{2 r-1}(s) \mathrm{d} s,
\end{aligned}
$$

where $e_{1}, \ldots, e_{k+1}$ is the canonical basis of $\mathbb{R}^{k+1}$ and $X_{m, n, r-\frac{1}{2}}(t)$ is the martingale given by (16).

(ii) Assume that for each $r, j \geq 1$,

$$
\sup _{n} \mathbb{E}\left(M_{m(n), n, r}^{j}(0)\right)=c(r, j)<\infty
$$

Then, for any $k \geq 0$ and $T>0$, there exists a positive constant $K\left(\underline{r}_{k+1}, T\right)$ such that

$$
\left.\sup _{n} \mathbb{E}\left(\sup _{0 \leq t \leq T} r_{\underline{i}_{k+1}^{m}}^{m(n), n}(t)\right)\right) \leq K\left(\underline{r}_{k+1}, T\right)<\infty .
$$

In particular, for every $r, j \geq 1$ and $T>0$, there exist a positive constant $K(r, j, T)$ such that

$$
\sup _{n} \mathbb{E}\left(\sup _{0 \leq t \leq T} M_{m(n), n, r}^{j}(t)\right) \leq K(r, j, T)<\infty .
$$

(iii) If for every $r \geq 1$

$$
M_{m(n), n, r}(0) \stackrel{\text { a.s. }}{\longrightarrow} 0 \text { as } n \rightarrow \infty,
$$

then

$$
\sup _{0 \leq t \leq T}\left|M_{m(n), n, r}(t)-\mu_{c, r}(t)\right| \stackrel{\text { a.s. }}{\longrightarrow} 0 \text { as } n \rightarrow \infty .
$$

Moreover if (20) is satisfied, then

$$
\mathbb{E}\left(\sup _{0 \leq t \leq T}\left|M_{m(n), n, r}(t)-\mu_{c, r}(t)\right|^{j}\right) \longrightarrow 0 \text { as } n \rightarrow \infty .
$$


Proof. (i) An application of Itô's formula to (2) gives that for $f \in C^{2}$,

$$
\begin{aligned}
&\left\langle\mu_{t}^{(m(n), n)}, f\right\rangle=\left\langle\mu_{0}^{(m(n), n)}, f\right\rangle \\
&+\frac{1}{n^{\frac{3}{2}}} \sum_{j=1}^{n} \int_{0}^{t} f^{\prime}\left(\lambda_{j}^{(m(n), n)}(s)\right) \sqrt{2 \lambda_{j}^{(m(n), n)}(s)} \mathrm{d} W_{j}^{(m(n), n)}(s) \\
&+\frac{1}{n} \sum_{j=1}^{n} \int_{0}^{t} f^{\prime \prime}\left(\lambda_{j}^{(m(n), n)}(s)\right) \lambda_{j}^{(m(n), n)}(s) \mathrm{d} s \\
&+\frac{m(n)}{n} \int_{0}^{t}\left\langle\mu_{s}^{(m(n), n)}, f^{\prime}\right\rangle \mathrm{d} s \\
&+\frac{1}{2} \int_{0}^{t} \mathrm{~d} s \int_{\mathbb{R}^{2}} \frac{\left[f^{\prime}(x)-f^{\prime}(y)\right](x+y)}{x-y} \mu_{s}^{(m(n), n)}(\mathrm{d} x) \mu_{s}^{(m(n), n)}(\mathrm{d} y), t \geq 0 .
\end{aligned}
$$

The relation (18) follows from (26) with $f(x)=x^{r}$.

Define $F: \mathbb{R}^{k+1} \longrightarrow \mathbb{R}$ by

$$
F\left(y_{1}, \ldots, y_{k+1}\right)=\prod_{r=1}^{k+1} y_{r}^{i_{r}}
$$

The Itô formula and (18) imply

$$
\begin{array}{rl}
\mathrm{d} r_{\underline{i}_{k+1}}^{m, n}(t) & =\mathrm{d} F\left(M_{m, n, 1}(t) \ldots . M_{m, n, k+1}(t)\right) \\
= & \sum_{r=1}^{k+1} \frac{\partial F\left(M_{m, n, 1}(t) \ldots M_{m, n, k+1}(t)\right)}{\partial y_{r}} \mathrm{~d} M_{m, n, r}(t) \\
& +\frac{1}{2} \sum_{l, r=1}^{k+1} \frac{\partial^{2} F\left(M_{m, n, 1}(t) \ldots M_{m, n, k+1}(t)\right)}{\partial y_{l} \partial y_{r}} \mathrm{~d}\left\langle M_{m, n, l}, M_{m, n, r}\right\rangle_{t} \\
=\sum_{r=1}^{k+1} & r i_{r} r_{\underline{i}_{k+1}-e_{r}}^{m, n}(t) \mathrm{d} X_{m, n, r-\frac{1}{2}}(t)+\frac{m}{n} \sum_{r=1}^{k+1} r i_{r} r_{\underline{i}_{k+1}-e_{r}}^{m, n}(t) M_{m, n, r-1}(t) \mathrm{d} t \\
& +\frac{1}{n} \sum_{r=1}^{k+1} r(r-1) i_{r} r_{\underline{i}_{k+1}-e_{r}}^{m, n}(t) M_{m, n, r-1}(t) \mathrm{d} t \\
& +\sum_{r=2}^{k+1} \sum_{l=0}^{r-2} r i_{r} r_{\underline{i}_{k+1}-e_{r}}^{m, n}(t) M_{m, n, r-1-l}(t) M_{m, n, l}(t) \mathrm{d} t \\
& +\frac{1}{n^{2}} \sum_{r=2}^{k+1} \sum_{l=1}^{r-1} l r i_{l} i_{r} r_{\underline{i}_{k+1}-e_{l}-e_{r}}^{m, n}(t) M_{m, n, l+r-1}(t) \mathrm{d} t \\
& +\frac{1}{2 n^{2}} \sum_{r=1}^{k+1} r^{2} i_{r}\left(i_{r}-1\right) r_{\underline{i}_{k+1}-2 e_{r}}^{m, n}(t) M_{m, n, 2 r-1}(t) \mathrm{d} t
\end{array}
$$




$$
\begin{aligned}
& =\sum_{r=1}^{k+1} r i_{r} r_{\underline{i}_{k+1}}^{m, n}(t) \mathrm{d} X_{m, n, r-\frac{1}{2}}(t)+\frac{m}{n} \sum_{r=1}^{k+1} r i_{r} r_{\underline{i}_{k+1}-e_{r}+e_{r-1}}^{m, n}(t) \mathrm{d} t \\
& +\frac{1}{n} \sum_{r=2}^{k+1} r(r-1) i_{r} r_{i_{k+1}-e_{r}+e_{r-1}}^{m, n}(t) \mathrm{d} t+\sum_{r=2}^{k+1} \sum_{l=1}^{r-2} r i_{r} r_{\underline{i}_{k+1}-e_{r}+e_{r-1-l}+e_{l}}^{m, n}(t) \mathrm{d} t \\
& +\frac{1}{n^{2}} \sum_{1 \leq l<r} l r i_{l} i_{r} r_{i_{k+1}-e_{l}-e_{r}+e_{l+r-1}}^{m, n}(t) \mathrm{d} t+\frac{1}{2 n^{2}} \sum_{r=1}^{k+1} r^{2} i_{r}\left(i_{r}-1\right) r_{\underline{i}_{k+1}-2 e_{r}+e_{2 r-1}}^{m, n}(t) \mathrm{d} t
\end{aligned}
$$

(ii) From (19) we obtain by induction over $k$ the estimate

$$
\left.\sup _{n} \sup _{0 \leq t \leq T} \mathbb{E}\left(r_{\underline{i}_{k+1}}^{m(n), n}(t)\right)\right) \leq K_{1}\left(\underline{r}_{k+1}, T\right)<\infty,
$$

and in particular

$$
\sup _{n} \sup _{0 \leq t \leq T} \mathbb{E}\left(M_{m(n), n, r}^{j}(t)\right) \leq K_{1}(r, j, T)<\infty .
$$

Next, from Burkholder's inequality and (28) we obtain

$$
\begin{aligned}
\mathbb{E}\left(\sup _{0 \leq t \leq T}\left|X_{m(n), n, r-\frac{1}{2}}(t)\right|^{k}\right) & \leq \frac{K(T, r, k)}{n^{k}} \mathbb{E}\left(\left[\int_{0}^{T} M_{m(n), n, 2 r-1}(s) \mathrm{d} s\right]^{\frac{k}{2}}\right) \\
& \leq \frac{K_{1}(T, r, k)}{n^{k}} \int_{0}^{T} \mathbb{E}\left(M_{m(n), n, 2 r-1}^{\frac{k}{2}}(s)\right) \mathrm{d} s \\
& \leq \frac{K_{1}(T, r, k)}{n^{k}} \int_{0}^{T}\left[\mathbb{E}\left(M_{m(n), n, 2 r-1}^{\left[\frac{k}{2}\right]+1}(s)\right)\right]^{\frac{\frac{k}{k}}{\left.\frac{k}{2}\right]+1}} \mathrm{~d} s \\
& \leq \frac{K_{2}(T, r, k)}{n^{k}} \leq K_{2}(T, r, k)<\infty .
\end{aligned}
$$

Now (21) (in particular (22)) follows easily from (19), by using (20), (28) and (29).

(iii) Now (29) implies

$$
\mathbb{E}\left(\sup _{0 \leq t \leq T}\left|X_{m(n), n, r-\frac{1}{2}}(t)\right|^{k}\right) \longrightarrow 0 \text {, as } n \rightarrow \infty .
$$

On the other hand, using Chebyshev inequality and (29) we have that for each $\varepsilon>0$

$$
\begin{aligned}
\sum_{n} \mathbb{P}\left(\sup _{0 \leq t \leq T}\left|X_{m(n), n, r-\frac{1}{2}}(t)\right|>\varepsilon\right) & \leq \frac{1}{\varepsilon^{2}} \sum_{n} \mathbb{E}\left(\sup _{0 \leq t \leq T}\left|X_{m(n), n, r-\frac{1}{2}}(t)\right|^{2}\right) \\
& \leq K_{2}(T, r, 2) \sum_{n} \frac{1}{n^{2}}<\infty,
\end{aligned}
$$

and thus

$$
\sup _{0 \leq t \leq T}\left|X_{m(n), n, r-\frac{1}{2}}(t)\right| \stackrel{\text { a.s. }}{\longrightarrow} 0 \text { as } n \rightarrow \infty .
$$

The almost surely convergence in (24) follows from (31) and (18) by an induction argument, since the family $\left\{\mu_{c, r}(t)\right\}_{t \geq 0, r \geq 1}$ satisfies uniquely the relation (14). 
Finally, (22) and (24) yield (25).

Remark 3.2. (a) The recurrence relation (18) involves products of previous moment processes and therefore it cannot be used to estimate or to compute the expectation of the moments. For this reason we need to work with the products $r_{\underline{i}_{k+1}}^{m, n}(t)$ which are stable by an application of the Itô formula.

(b) Define the vector-valued process

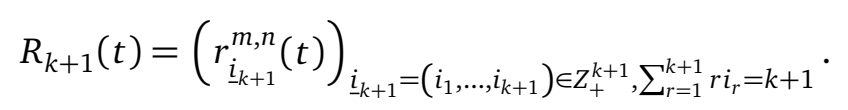

The proof of Theorem 3.1 implies the existence of a deterministic matrix $D_{k}$ such that

$$
\mathbb{E}\left(R_{k+1}(t)\right)=\mathbb{E}\left(R_{k+1}(0)\right)+D_{k} \int_{0}^{t} \mathbb{E}\left(R_{k}(s)\right) \mathrm{d} s .
$$

The inductive relation (32) provides in particular the explicit computation of the moments $M_{m, n, r}(t)$; see [14] for real Wishart distributions, [13], [22] for complex Wishart distributions and [15] for the case of real Wishart processes.

Theorem 3.3. For any $T>0$ and any bounded continuous function $f: \mathbb{R} \longrightarrow \mathbb{R}$ we have

$$
\lim _{n \rightarrow \infty} \sup _{0 \leq t \leq T}\left|\int f(x) \mu_{t}^{(m(n), n)}(\mathrm{d} x)-\int f(x) \mu_{c}(t)(\mathrm{d} x)\right|=0 \text { a.s. }
$$

That is, the empirical process $\left\{\left(\mu_{t}^{(m(n), n)}\right)_{t \geq 0}\right\}_{n \geq 1}$ converges a.s. in the space $C\left(\mathbb{R}_{+}, \operatorname{Pr}(\mathbb{R})\right)$.

Proof. From Theorem 3.1 follows the convergence (33) for continuous functions with compact support.

Now we prove the tightness of the sequence of processes $\left\{\left(\mu_{t}^{(m(n), n)}\right)_{t \geq 0}\right\}_{n \geq 1}$ in the space $C\left(\mathbb{R}_{+}, \operatorname{Pr}(\mathbb{R})\right)$.

From (26) and (29) it is easily seen that for every $0 \leq t_{1} \leq t_{2} \leq T, n \geq 1$ and $f \in C_{b}^{2}(\mathbb{R})$,

$$
\mathbb{E}\left(\left|\left\langle\mu_{t_{1}}^{(m(n), n)}, f\right\rangle-\left\langle\mu_{t_{2}}^{(m(n), n)}, f\right\rangle\right|^{4}\right) \leq K(T, f)\left|t_{1}-t_{2}\right|^{2},
$$

which, by the well known standard criterion, shows that the sequence of continuous real processes $\left\{\left\langle\mu_{t}^{(m(n), n)}, f\right\rangle_{t \geq 0}\right\}_{n \geq 1}$ is tight and consequently the sequence of processes $\left\{\left(\mu_{t}^{(m(n), n)}\right)_{t \geq 0}\right\}_{n \geq 1}$ is tight in the space $C\left(\mathbb{R}_{+}, \operatorname{Pr}(\mathbb{R})\right)$ (see [10, pp. 107]).

Then, for any $\varepsilon, T>0$ there exists a compact $K_{\varepsilon, T} \subset C([0, T], \operatorname{Pr}(\mathbb{R}))$ such that

$$
\sup _{n} \mathbb{P}\left(\mu^{(m(n), n)} \in C K_{\varepsilon, T}\right) \leq \varepsilon
$$

In particular we find a compact $\Gamma_{\varepsilon, T} \subset \operatorname{Pr}(\mathbb{R})$ such that

$$
K_{\varepsilon, T} \subset\left\{\mu: \mu_{t} \in \Gamma_{\varepsilon, T}, \forall 0 \leq t \leq T\right\}
$$


and consequently, there exists $a_{\varepsilon, T}>0$ such that

$$
\begin{gathered}
\sup _{\mu \in K_{\varepsilon, T}} \sup _{0 \leq t \leq T} \mu_{t}\left(|x| \geq a_{\varepsilon, T}\right) \leq \varepsilon \\
\sup _{0 \leq t \leq T} \mu_{c}(t)\left(|x| \geq a_{\varepsilon, T}\right) \leq \varepsilon
\end{gathered}
$$

(in fact we have $\sup _{0 \leq t \leq T} \mu_{c}(t)\left(|x| \geq a_{\varepsilon, T}\right)=0$ for $a_{\varepsilon, T}$ enough large).

From (34), (35) and an approximation argument we obtain (33) for all continuous and bounded functions.

Corollary 3.4. For any interval $(a, b) \subset \mathbb{R}$

$$
\lim _{n \rightarrow \infty} \max _{0 \leq t \leq T}\left|\frac{1}{n} \#\left\{1 \leq j \leq n: \lambda_{j}^{(m(n), n)}(t) \in[a, b]\right\}-\mu_{c}(t)([a, b])\right|=0 \text { a.s. }
$$

As a consequence of the above theorem we obtain Wachter's result on almost sure convergence of the empirical distribution of the eigenvalues to the measures $\mu_{c}^{f p}$ (see [11], [28]), since the moments determine uniquely the free Poisson law and also the well known result (taking $t=1$ ) on the weak convergence of traces of complex Wishart matrices [11, Theorem 6.7].

In the case of Dyson Brownian motion the limit in the previous theorem is the family of semicircle laws which agrees with the dilations of the standard semicircle law (see [5], [16], [25]). However, in the case of Laguerre process, the limit is the family of dilations of free Poisson laws which is different from the family of free Poisson laws, obtained by taking $c t$ as the parameter, which correspond to the free Poisson Lévy process.

\subsection{Large deviations approach}

The result of Theorem 3.3 is also a consequence of the following large deviations upper bound for the empirical process with good rate function (see [5, Theorem 3.2 and Corollary 3.1]).

Denote $\tilde{C}_{b}^{1,2}\left([0, T] \times \mathbb{R}_{+}\right)$the subset of $C_{b}^{1,2}\left([0, T] \times \mathbb{R}_{+}\right)$of functions $f$ such that

$$
\sup _{0 \leq t \leq T} \sup _{s \in \mathbb{R}_{+}}\left|x \frac{\partial^{2} f(t, x)}{\partial x^{2}}\right|<\infty, \sup _{0 \leq t \leq T} \sup _{x \in \mathbb{R}_{+}}\left|x\left(\frac{\partial f(t, x)}{\partial x}\right)^{2}\right|<\infty .
$$

For any $0 \leq s<t \leq T, f, g \in \tilde{C}_{b}^{1,2}\left([0, T] \times \mathbb{R}_{+}\right), v \in C\left([0, T], \operatorname{Pr}\left(\mathbb{R}_{+}\right)\right)$with

$$
\sup _{0 \leq t \leq T} \int x v_{t}(\mathrm{~d} x)<\infty
$$


define,

$$
\begin{aligned}
& S^{s, t}(v, f)=\int f(t, x) v_{t}(\mathrm{~d} x)-\int f(s, x) v_{s}(\mathrm{~d} x) \\
&-\int_{s}^{t} \int \frac{\partial f(u, x)}{\partial u} v_{u}(\mathrm{~d} x) \mathrm{d} u-c \int_{s}^{t} \int \frac{\partial f(u, x)}{\partial x} v_{u}(\mathrm{~d} x) \mathrm{d} u \\
&-\frac{1}{2} \int_{s}^{t} \iint \frac{\left(\frac{\partial f(u, x)}{\partial x}-\frac{\partial f(u, y)}{\partial x}\right)(x+y)}{x-y} v_{u}(\mathrm{~d} x) v_{u}(\mathrm{~d} y) \mathrm{d} u, \\
&\langle f, g\rangle_{v}^{s, t}=\int_{s}^{t} \int x \frac{\partial f(u, x)}{\partial x} \frac{\partial g(u, x)}{\partial x} v_{u}(\mathrm{~d} x) \mathrm{d} u, \\
& \bar{S}^{s, t}(v, f)=S^{s, t}(v, f)-2\langle f, g\rangle_{v}^{s, t}, \\
& S(v)=\left\{\begin{array}{c}
\infty, \\
\sup _{0 \leq s<t \leq T} v_{f \in \tilde{C}_{b}^{1,2}\left([0,1] \times \mathbb{R}_{+}\right)} \bar{s}^{s, t}(v, f), \quad \delta_{0}=\delta_{0} .
\end{array}\right.
\end{aligned}
$$

The sequence $\left\{\left(\mu_{t}^{(m(n), n)}\right)_{t \in[0, T]}\right\}_{n \geq 1}$ satisfies in the space $C\left([0, T], \operatorname{Pr}\left(\mathbb{R}_{+}\right)\right)$the large deviation upper bound with good rate function $S$ :

$$
\varlimsup_{n \rightarrow \infty} \frac{1}{n^{2}} \ln \mathbb{P}\left(\mu^{(m(n), n)} \in F\right) \leq-\inf _{v \in F} S(v)
$$

for every closed subset $F \subset C\left([0, T], \operatorname{Pr}\left(\mathbb{R}_{+}\right)\right)$.

In particular the sequence $\left\{\left(\mu_{t}^{(m(n), n)}\right)_{t \in[0, T]}\right\}_{n \geq 1}$ is exponentially tight.

Proposition 3.5. Let $v \in C\left([0, T], \operatorname{Pr}\left(\mathbb{R}_{+}\right)\right)$be such that for some $\varepsilon_{0}>0$,

$$
\sup _{0 \leq t \leq T} \int x^{1+\varepsilon_{0}} v_{t}(\mathrm{~d} x)<\infty .
$$

Then

$$
\lim _{\substack{r \rightarrow \infty \\ \varepsilon \rightarrow 0}} \varlimsup_{n \rightarrow \infty} \frac{1}{n^{2}} \ln \mathbb{P}\left(\sup _{0 \leq t \leq T}\left|M_{m(n), n, k}(t)-\int x^{k} v_{t}(\mathrm{~d} x)\right| \leq \varepsilon, k \leq r\right) \leq-S(v) .
$$

Proof. If we apply (37) to the closed set $F(\varepsilon, r)=\bar{A}(\varepsilon, r)$, where

$$
A(\varepsilon, r)=\left\{\mu \in C([0, T], \operatorname{Pr}(\mathbb{R})): \mid \int x^{k} \mu_{t}(\mathrm{~d} x)-\int x^{k} v_{t}(\mathrm{~d} x \mid \leq \varepsilon, k \leq r\},\right.
$$

we obtain for every $\varepsilon, r>0$,

$$
\varlimsup_{n \rightarrow \infty} \frac{1}{n^{2}} \ln \mathbb{P}\left(\mu^{(m(n), n)} \in F(\varepsilon, r)\right) \leq-\inf _{\mu \in F(\varepsilon, r)} S(\mu) .
$$


Next, let $\varepsilon_{j} \longrightarrow 0, r_{j} \longrightarrow \infty$ and choose $\mu^{k(j)} \in A\left(\varepsilon_{j}, r_{j}\right), j \geq 1$, such that

$$
\inf _{\mu \in F\left(\varepsilon_{j}, r_{j}\right)} S(\mu)=S\left(\mu^{k(j)}\right)
$$

Then it is easily seen (like in the proof of Theorem 3.3) that $\mu^{k(j)}$ converges to $\mu$ in $C\left([0, T], \operatorname{Pr}\left(\mathbb{R}_{+}\right)\right)$and conclude by the lower semicontinuity of $S$.

\subsection{Fluctuations of moments processes}

In this section we consider the asymptotic fluctuations of the moments processes $\left\{M_{m(n), n, r}(t)\right\}_{t \geq 0}$ around the corresponding moments $\left\{\mu_{c, r}(t)\right\}_{t \geq 0}$ of the dilations of free Poisson distribution. Let

$$
Y_{t}^{(n)}=n\left(\mu_{t}^{(m(n), n)}-\mu_{c}(t)\right) \text {, }
$$

$V_{n, 0}(t)=0$ and for $r \geq 1$

$$
V_{n, r}(t)=\int x^{r} Y_{t}^{(n)}(\mathrm{d} x)=n\left(M_{m(n), n, r}(t)-\mu_{c, r}(t)\right)
$$

From (26) we obtain that for $f \in C^{2}$ and $t \geq 0$

$$
\begin{aligned}
&\left\langle Y_{t}^{(n)}, f\right\rangle=\left\langle Y_{0}^{(n)}, f\right\rangle \\
&+\frac{1}{\sqrt{n}} \sum_{j=1}^{n} \int_{0}^{t} f^{\prime}\left(\lambda_{j}^{(m(n), n)}(s)\right) \sqrt{2 \lambda_{j}^{(m(n), n)}(s)} \mathrm{d} W_{j}^{(m(n), n)}(s) \\
&+\frac{1}{\sqrt{n}} \sum_{j=1}^{n} \int_{0}^{t} f^{\prime}\left(\lambda_{j}^{(m(n), n)}(s)\right) \sqrt{2 \lambda_{j}^{(m(n), n)}(s)} \mathrm{d} W_{j}^{(m(n), n)}(s) \\
&+\frac{m(n)}{n} \int_{0}^{t}\left\langle Y_{s}^{(n)}, f^{\prime}\right\rangle \mathrm{d} s+\left(\frac{m(n)}{n}-c\right) \int_{0}^{t}\left\langle\mu_{c}(s), f^{\prime}\right\rangle \mathrm{d} s \\
&+\frac{1}{n} \sum_{j=1}^{n} \int_{0}^{t} f^{\prime \prime}\left(\lambda_{j}^{(m(n), n)}(s)\right) \lambda_{j}^{(m(n), n)}(s) \mathrm{d} s \\
&+\frac{1}{2} \int_{0}^{t} \mathrm{~d} s \int_{\mathbb{R}^{2}} \frac{\left(f^{\prime}(x)-f^{\prime}(y)\right)(x+y)}{x-y}\left[\left(\mu_{s}^{(m(n), n)}(\mathrm{d} x)+\mu_{c}(s)(\mathrm{d} x)\right) Y_{s}^{(n)}(\mathrm{d} y)\right] .
\end{aligned}
$$

The martingales

$$
N_{n, r}(t)=\frac{1}{\sqrt{n}} \sum_{j=1}^{n} \int_{0}^{t}\left[\lambda_{j}^{(m(n), n)}(s)\right]^{r} \mathrm{~d} W_{j}^{(m(n), n)}(s), \quad t \geq 0, r \geq 1,
$$

play an important role (see [25] for the Dyson Brownian model).

Next, for a $r$-dimensional martingale $C=\left(C_{j}: 1 \leq j \leq r\right)$ denote by $[C, C]$ the $\mathbb{R}^{r} \otimes \mathbb{R}^{r}$-valued process whose components are the quadratic variations $\left[C_{j}, C_{k}\right]_{1 \leq j, k \leq r}$. 
Proposition 3.6. Assume (20) holds. Then, for each $r \geq 1$ the $r$-dimensional martingale $\left(N_{n, k-\frac{1}{2}}\right)_{1 \leq k \leq r}$ converges weakly in $C\left(\mathbb{R}_{+}, \mathbb{R}^{r}\right)$, when $n$ goes to infinity, to a continuous centered Gaussian martingale $\left(N_{k-\frac{1}{2}}\right)_{1 \leq k \leq r}$ with

$$
\mathbb{E}\left(N_{k-\frac{1}{2}}(s) N_{l-\frac{1}{2}}(t)\right)=\frac{\mu_{c, k+l-1}(1)}{k+l}(s \wedge t)^{k+l}
$$

and quadratic variation

$$
\left[N_{k-\frac{1}{2}}, N_{l-\frac{1}{2}}\right]_{t}=\frac{\mu_{c, k+l-1}(1)}{k+l} t^{k+l}
$$

Proof. Using the equality

$$
\left[N_{n, k-\frac{1}{2}}, N_{n, l-\frac{1}{2}}\right]_{t}=\int_{0}^{t} M_{m(n), n, k+l-1}(s) \mathrm{d} s,
$$

Burkholder's inequality and (22), we obtain for each $0 \leq t_{1} \leq t_{2} \leq T$,

$$
\begin{aligned}
\mathbb{E}\left(\left|N_{n, k-\frac{1}{2}}\left(t_{2}\right)-N_{n, k-\frac{1}{2}}\left(t_{1}\right)\right|^{4}\right) & \leq K_{1} \mathbb{E}\left(\left|\int_{t_{1}}^{t_{2}} M_{m(n), n, 2 k-1}(s) \mathrm{d} s\right|^{2}\right) \\
& \leq K_{1}\left(t_{2}-t_{1}\right) \int_{t_{1}}^{t_{2}} \mathbb{E}\left(M_{m(n), n, 2 k-1}^{2}(s)\right) \mathrm{d} s \\
& \leq K_{2}\left(t_{2}-t_{1}\right)^{2}
\end{aligned}
$$

and thus the sequence $\left(N_{n, k-\frac{1}{2}}: 1 \leq k \leq r\right)$ is tight in $C\left(\mathbb{R}_{+}, \mathbb{R}^{r}\right)$.

Choose a subsequence $N^{j}:=\left(N_{n(j), k-\frac{1}{2}}: 1 \leq k \leq r\right)_{j \geq 1}$ which converges weakly to a limit $N=$ $\left(N_{k-\frac{1}{2}}\right)_{1 \leq k \leq r}$.

By [18, Corollary 1.19, pp. 486] It follows that $N$ is a continuous $r$-dimensional local martingale (in fact it is a martingale) and by [18, Theorem 6.1] we have that $\left(N^{j},\left[N^{j}, N^{j}\right]\right)$ converges weakly to $(N,[N, N])$ in $C\left(\mathbb{R}_{+}, \mathbb{R}^{r} \times\left(\mathbb{R}^{r} \otimes \mathbb{R}^{r}\right)\right)$.

In particular $\left[N^{j}, N^{j}\right]$ converges weakly to $[N, N]$. From (13), (24) and (44) we deduce that $\left[N^{j}, N^{j}\right]_{t}$ converges almost surely to

$$
\left(\int_{0}^{t} \mu_{c, k+l-1}(s) \mathrm{d} s\right)_{1 \leq k, l \leq r}=\left(\frac{\mu_{c, k+l-1}(1)}{k+l} t^{k+l}\right)_{1 \leq k, l \leq r}
$$

and thus

$$
[N, N]_{t}=\left(\frac{\mu_{c, k+l-1}(1)}{k+l} t^{k+l}\right)_{1 \leq k, l \leq r} .
$$

By [18, Theorem 4.15] $N$ is a process with independent increments and for $s<t$ the random variable $N_{t}-N_{s}$ is has centered Gaussian distribution. Therefore $N$ is a continuous centered Gaussian martingale which satisfies (42) and (43). 
In the final result of this section we show for each $r \geq 1$, the fluctuation processes $V_{n, r}$ converge weakly to a one dimensional Gaussian process $Z_{r}$, which is given by a recursive expression that involves $Z_{1}, \ldots, Z_{r-1}$, the Gaussian martingale $N_{r-\frac{1}{2}}$ and the families of moments $\left\{\mu_{c, k}(t)\right\}_{t \geq 0}, k=$ $1, \ldots, r-1$.

Theorem 3.7. For every $k \geq 1$ assume that $V_{n, k}(0)$ converges weakly to $V_{k}^{(0)} \in \mathbb{R}$ as $n \rightarrow \infty$ and (20). Then, for each $r \geq 1$, the $r$-dimensional process $\left(V_{n, k}\right)_{1 \leq k \leq r}$ converges weakly in $C\left(\mathbb{R}_{+}, \mathbb{R}^{r}\right)$ to the unique in law continuous Gaussian process $\left(Z_{k}\right)_{1 \leq k \leq r}$ given by

$$
\begin{gathered}
Z_{k}(t)=V_{k}^{(0)}+k \sqrt{2} N_{k-\frac{1}{2}}(t) \\
+k\left(c+\frac{1}{2}\right) \int_{0}^{t} Z_{k-1}(s) \mathrm{d} s+\mu_{c, k-1}(1) t^{k} \\
+2 k \int_{0}^{t}\left[\mu_{c, k-2}(s) Z_{1}(s)+\ldots+\mu_{c, 1}(s) Z_{k-2}(s)\right] \mathrm{d} s, 1 \leq k \leq r,
\end{gathered}
$$

where $Z_{0}=0$ and $N_{k-\frac{1}{2}}$ is given by (41).

Proof. From (45) and by induction on $k$ it is easily seen that

$$
\begin{aligned}
Z_{k}(t) & =P_{k}(t)+k \sqrt{2} N_{k-\frac{1}{2}}(t)+c \sum_{j=1}^{k-1} \int_{0}^{t} s_{1}^{j_{1}} N_{j-\frac{1}{2}}\left(s_{1}\right) \mathrm{d} s_{1} \\
& +\sum_{j=1}^{k-2} c_{j} \int_{0}^{t} \int_{0}^{s_{1}} . . \int_{0}^{s_{j}} s_{1}^{r_{1}} \ldots s_{j+1}^{r_{j+1}} \sum_{i=1}^{j} N_{i-\frac{1}{2}}\left(s_{j+1}\right) \mathrm{d} s_{j+1} \ldots \mathrm{d} s_{1},
\end{aligned}
$$

where $P_{k}(t)$ is a polynomial of order $k, c, c_{j}>0$ and $j_{1}, r_{1}, \ldots, r_{j+1} \geq 0$.

It is clear that (46) implies that the distribution of the process $\left(Z_{k}\right)_{1 \leq k \leq r}$ is uniquely determined by the distribution of the $k$-dimensional Gaussian martingale $\left(N_{k-\frac{1}{2}}\right)_{1 \leq k \leq r}$. In particular $\left(Z_{k}\right)_{1 \leq k \leq r}$ is a Gaussian process.

Taking $f(x)=x^{k}$ in (40) we obtain the equality

$$
\begin{gathered}
V_{n, k}(t)=V_{n, k}(0)+k \sqrt{2} N_{n, k-\frac{1}{2}}(t) \\
+k\left(\frac{m(n)}{n}+\frac{k-1}{n}+\frac{1}{2}\right) \int_{0}^{t} V_{n, k-1}(s) \mathrm{d} s \\
+\left(\frac{m(n)}{n}-c\right) k \int_{0}^{t} \mu_{c, k-1}(s) \mathrm{d} s+\frac{k}{2} \int_{0}^{t}\left[M_{m(n), n, k-1}(s)+\mu_{c, k-1}(s)\right] \mathrm{d} s \\
+k \int_{0}^{t}\left\{\left[M_{m(n), n, k-2}(s)+\mu_{c, k-2}(s)\right] V_{n, 1}(s)+\left[M_{m(n), n, 1}(s)+\mu_{c, 1}(s)\right] V_{n, k-2}(s)\right\} \mathrm{d} s .
\end{gathered}
$$

By the Skorohod representation of the weak convergence (eventually in a new probability space) we can assume that

$$
\left(V_{n, k}(0)_{1 \leq k \leq r},\left(N_{n, k-\frac{1}{2}}\right)_{1 \leq k \leq r}\right)
$$


converges a.s. in $\mathbb{R}^{r} \times C\left(\mathbb{R}_{+}, \mathbb{R}^{r}\right)$ to

$$
\left(\left(V_{k}^{(0)}\right)_{1 \leq k \leq r},\left(N_{k-\frac{1}{2}}\right)_{1 \leq k \leq r}\right) .
$$

Then, by induction we deduce that $\left(V_{n, k}\right)_{1 \leq k \leq r}$ defined by (47) converges a.s. to $\left(Z_{k}\right)_{1 \leq k \leq r}$ given by (45).

\section{Convergence of extreme eigenvalues processes}

The behavior of the largest and smallest eigenvalues of Wishart random matrices was established in [1], [2], [3], [12], [26] (see also [11] for a more recent proof). In the next theorem we extend these results for the supremum of the largest eigenvalue process as well as for the infimum of the smallest eigenvalue process from a Laguerre process.

Theorem 4.1. Assume that

$$
\sup _{n} \operatorname{Tr}\left(\sum m(n), n(0)\right)=\sup _{n}\left(\lambda_{1}^{(m(n), n)}(0)+\ldots+\lambda_{n}^{(m(n), n)}(0)\right)<\infty .
$$

Then for each $T>0$ we have

$$
\begin{aligned}
& \lim _{n \rightarrow \infty} \max _{0 \leq t \leq T} \lambda_{n}^{(m(n), n)}(t) \stackrel{\text { a.s. }}{\longrightarrow}(1+\sqrt{c})^{2} T, \\
& \lim _{n \rightarrow \infty} \min _{0 \leq t \leq T} \lambda_{1}^{(m(n), n)} \stackrel{\text { a.s. }}{\longrightarrow}(1-\sqrt{c})^{2} T .
\end{aligned}
$$

To prove this theorem, we need the next lemmas. Denote $c_{n}=\frac{m(n)}{n}$.

Lemma 4.2. Assume that (48) is satisfied. Then, for every $T>0, \alpha \in\left[0, \frac{n}{4 T}\right]$, the following estimations hold

$$
\begin{gathered}
\mathbb{E}\left[\exp \left(\alpha \lambda_{n}^{(m(n), n)}(T)\right)\right] \leq C(T) n^{\frac{1}{2}} \exp \left(\alpha T k+\frac{2 \alpha^{2} T^{2}\left(1+c_{n}\right)}{n}\right), \\
\mathbb{E}\left[\exp \left(\alpha \int_{0}^{T} \lambda_{n}^{(m(n), n)}(s) \mathrm{d} s\right)\right] \leq C(T) n^{\frac{1}{2}} \exp \left(\frac{\alpha T^{2}}{2} k_{n}+\frac{2 \alpha^{2} T^{4}}{3 n}\left(1+c_{n}\right)\right),
\end{gathered}
$$

for some positive constant $C(T)$, where $k_{n}=\left(1+\sqrt{c_{n}}\right)^{2}$.

Proof. Suppose first the centered case, that is $B_{m(n), n}(0)=0$.

From the following estimate (see [11, inequality 7.13])

$$
\begin{aligned}
& \mathbb{E}\left[\exp \left(\alpha \lambda_{n}^{(m(n), n)}(1)\right)\right] \leq \mathbb{E}\left[\operatorname{Tr}\left(\exp \left(\alpha \sum_{m(n), n}(1)\right)\right)\right] \\
& \leq n \exp \left(\left(1+\sqrt{c_{n}}\right)^{2} \alpha+\frac{\left(1+c_{n}\right) \alpha^{2}}{n}\right), \forall \alpha \in\left[0, \frac{n}{2}\right],
\end{aligned}
$$


and the equality in law

$$
\lambda_{n}^{(m(n), n)}(t) \stackrel{L}{=} t \lambda_{n}^{(m(n), n)}(1)
$$

we obtain for each $\alpha \in\left[0, \frac{n}{2 T}\right]$

$$
\mathbb{E}\left[\exp \left(\alpha \lambda_{n}^{(m(n), n)}(T)\right)\right] \leq n \exp \left(\alpha T\left(1+\sqrt{c_{n}}\right)^{2}+\frac{\alpha^{2} T^{2}\left(1+c_{n}\right)}{n}\right) .
$$

Next, by using (53) and the generalized Hölder's inequality, we have

$$
\begin{gathered}
\mathbb{E}\left[\exp \left(\alpha \int_{0}^{T} \lambda_{n}^{(m(n), n)}(s) \mathrm{d} s\right)\right] \\
\leq \lim \inf _{k \rightarrow \infty} \mathbb{E}\left[\exp \left(\frac{\alpha T}{k} \sum_{r=0}^{k-1} \lambda_{n}^{(m(n), n)}\left(\frac{r T}{k}\right)\right)\right] \\
\leq \lim _{k \rightarrow \infty} \prod_{r=0}^{k-1}\left\{\mathbb{E}\left[\exp \left(\alpha T \lambda_{n}^{(m(n), n)}\left(\frac{r T}{k}\right)\right)\right]\right\}^{\frac{1}{k}} \\
\leq \lim \inf _{k \rightarrow \infty} \prod_{r=0}^{k-1}\left\{n \exp \left(\left(1+\sqrt{c_{n}}\right)^{2} \frac{\alpha r T^{2}}{k}+\frac{\left(1+c_{n}\right) \alpha^{2} r^{2} T^{4}}{k^{2}}\right)\right\}^{\frac{1}{k}} \\
=\lim \inf _{k \rightarrow \infty} n \exp \left(\left(1+\sqrt{c_{n}}\right)^{2} \frac{\alpha T^{2}}{k^{2}} \sum_{r=0}^{k-1} r+\frac{\left(1+c_{n}\right) \alpha^{2} T^{4}}{k^{3}} \sum_{r=0}^{k-1} r^{2}\right) \\
=n \exp \left(\frac{\alpha T^{2}}{2}\left(1+\sqrt{c_{n}}\right)^{2}+\frac{\alpha^{2} T^{4}}{3 n}\left(1+c_{n}\right)\right) \cdot
\end{gathered}
$$

In the noncentered case define

$$
\begin{gathered}
B_{m(n), n}^{(1)}(t)=B_{m(n), n}(t)-B_{m(n), n}(0) \\
\sum_{m(n), n}^{(1)}(t)=\frac{1}{2 n}\left(B_{m(n), n}^{(1)}(t)\right)^{*} B_{m(n), n}^{(1)}(t),
\end{gathered}
$$

and consider the following Laguerre process with drift

$$
\begin{gathered}
\sum_{m(n), n, T}^{(1)}(t)= \\
\frac{1}{2 n}\left(B_{m(n), n}^{(1)}(t)+\frac{t}{T} B_{m(n), n}(0)\right)^{*}\left(B_{m(n), n}^{(1)}(t)+\frac{t}{T} B_{m(n), n}(0)\right), 0 \leq t \leq T .
\end{gathered}
$$

Let $\lambda_{n}^{(1, m(n), n)}(t)$ (resp. $\left.\lambda_{n, T}^{(1, m(n), n)}(t)\right)$ be the largest eigenvalue of $\sum_{m(n), n}^{(1)}(t)$ (resp. $\left.\sum_{m(n), n, T}^{(1)}(t)\right)$. Note that $\lambda_{n, T}^{(1, m(n), n)}(T)=\lambda_{n}^{(m(n), n)}(T)$. 
If $G$ is a nonnegative measurable functional defined on the space of continuous functions, then by the multivariate Cameron-Martin formula we have the equality

$$
\begin{gathered}
\mathbb{E}\left[G\left(\sum_{m(n), n, T}^{(1)}(s), 0 \leq s \leq t\right)\right]=\mathbb{E}\left[G\left(\sum_{m(n), n}^{(1)}(s), 0 \leq s \leq t\right)\right. \\
\left.\exp \left\{-\frac{1}{T} \operatorname{Tr}\left(B_{m(n), n}(0)^{*} B_{m(n), n}^{(1)}(t)\right)-\frac{t}{2 T^{2}} \operatorname{Tr}\left(B_{m(n), n}(0)^{*} B_{m(n), n}(0)\right)\right\}\right],
\end{gathered}
$$

for any $0 \leq t \leq T$.

Applying (55) to $\exp \left(\alpha \lambda_{n, T}^{(1, m(n), n)}(t)\right)$ we get

$$
\begin{gathered}
\mathbb{E}\left[\exp \left(\alpha \lambda_{n, T}^{(1, m(n), n)}(t)\right)\right]=\mathbb{E}\left[\exp \left(\alpha \lambda_{n}^{(1, m(n), n)}(t)\right)\right. \\
\left.\exp \left\{-\frac{1}{T} \operatorname{Tr}\left(B_{m(n), n}(0)^{*} B_{m(n), n}^{(1)}(t)\right)-\frac{t}{2 T^{2}} \operatorname{Tr}\left(B_{m(n), n}(0)^{*} B_{m(n), n}(0)\right)\right\}\right] \\
\leq\left\{\mathbb{E}\left[\exp \left(2 \alpha \lambda_{n}^{(1, m(n), n)}(t)\right)\right]\right\}^{\frac{1}{2}} \times \\
\left\{\mathbb{E}\left[\exp \left\{-\frac{2}{T} \operatorname{Tr}\left(B_{m(n), n}(0)^{*} B_{m(n), n}^{(1)}(t)\right)-\frac{t}{T^{2}} \operatorname{Tr}\left(B_{m(n), n}(0)^{*} B_{m(n), n}(0)\right)\right\}\right]\right\}^{\frac{1}{2}} \\
\leq n^{\frac{1}{2}} \exp \left(\alpha t\left(1+\sqrt{c_{n}}\right)^{2}+\frac{2 \alpha^{2} t^{2}\left(1+c_{n}\right)}{n}\right) \times \\
\left\{\mathbb{E}\left[\exp \left\{-\frac{2}{T} \operatorname{Tr}\left(B_{m(n), n}(0)^{*} B_{m(n), n}^{(1)}(t)\right)-\frac{2 t}{T^{2}} \operatorname{Tr}\left(B_{m(n), n}(0)^{*} B_{m(n), n}(0)\right)\right\}\right]\right\}^{\frac{1}{2}} \\
\times \exp \left\{\frac{t}{T^{2}} \operatorname{Tr}\left(B_{m(n), n}(0)^{*} B_{m(n), n}(0)\right)\right\} \\
\leq \exp \left\{\frac{1}{T} \sup _{k} \operatorname{Tr}\left(B_{m(k), k}(0)^{*} B_{m(k), k}(0)\right)\right\} n^{\frac{1}{2}} \exp \left(\alpha t k_{n}+\frac{2 \alpha^{2} t^{2}\left(1+c_{n}\right)}{n}\right) .
\end{gathered}
$$

The estimate (52) follows from (51) as in the centered case.

Remark 4.3. By using the estimate (see $[11,7.14]$ )

$$
\mathbb{E}\left[\exp \left(-\alpha \lambda_{1}^{(m(n), n)}(1)\right)\right] \leq n \exp \left(-\left(\sqrt{c_{n}}-1\right)^{2} \alpha+\frac{\left(c_{n}+1\right) \alpha^{2}}{n}\right), \forall \alpha>0,
$$

in the centered case, in a similar manner it follows the inequality

$$
\begin{gathered}
\mathbb{E}\left[\exp \left(-\alpha \int_{0}^{T} \lambda_{1}^{(m(n), n)}(s) \mathrm{d} s\right)\right] \\
\leq C(T) n^{\frac{1}{2}} \exp \left(-\frac{\alpha T^{2}}{2}\left(1-\sqrt{c_{n}}\right)^{2}+\frac{2 \alpha^{2} T^{4}}{3 n}\left(1+c_{n}\right)\right), \forall \alpha>0 .
\end{gathered}
$$


Remark 4.4. The above argument based on the Cameron-Martin formula provides an alternative method for proving the boundedness of the moments in Theorem 3.1 (inequality (28) and consequently (22)).

Lemma 4.5. For $\alpha \in\left[0, \sqrt{\frac{n}{2 T}}\right]$, let

$$
X_{\max }^{\alpha}(t)=\exp \left(\alpha \int_{0}^{t} \sqrt{\lambda_{n}^{(m(n), n)}(s)} \mathrm{d} W_{n}^{(n)}-\frac{\alpha^{2}}{2} \int_{0}^{t} \lambda_{n}^{(m(n), n)}(s) \mathrm{d} s,\right)
$$

and for $\alpha>0$ let

$$
X_{\min }^{\alpha}(t)=\exp \left(-\alpha \int_{0}^{t} \sqrt{\lambda_{1}^{(m(n), n)}(s)} \mathrm{d} W_{1}^{(n)}-\frac{\alpha^{2}}{2} \int_{0}^{t} \lambda_{1}^{(m(n), n)}(s) \mathrm{d} s\right) .
$$

Then the processes $t \longrightarrow X_{\max }^{\alpha}(t), t \longrightarrow X_{\min }^{\alpha}(t)$ are martingales. In particular, the processes

$$
\begin{aligned}
& t \longrightarrow \exp \left(\alpha \lambda_{n}^{(m(n), n)}(t)\right), \alpha \in\left[0, \sqrt{\frac{n}{2 T}}\right], \\
& t \longrightarrow \exp \left(-\alpha \lambda_{1}^{(m(n), n)}(t)\right), \alpha>0,
\end{aligned}
$$

are submartingales.

Proof. The fact that (57) (and (58)) is a martingale follows from the previous lemma and Novikov's criterion.

Next, if $t_{1}<t_{2}$ from (2) and the fact that (57) is a martingale, we have

$$
\begin{gathered}
\mathbb{E}\left[\exp \left(\alpha \lambda_{n}^{(m(n), n)}\left(t_{2}\right)\right) \mid \sum_{m(n), n}(s): s \leq t_{1}\right] \\
=\mathbb{E}\left[\operatorname { e x p } \left\{\alpha \int_{0}^{t_{2}} \sqrt{\frac{2 \lambda_{n}^{(m(n), n)}(s)}{n}} \mathrm{~d} W_{n}^{(n)}(s)\right.\right. \\
\left.\left.+\frac{\alpha}{n} \int_{0}^{t_{2}}\left(m+\sum_{j=1}^{n-1} \frac{\lambda_{n}^{(m(n), n)}(s)+\lambda_{j}^{(n)}(s)}{\lambda_{n}^{(m(n), n)}(s)-\lambda_{j}^{(n)}(s)} \mathrm{d} s\right)\right\} \mid \sum_{m(n), n}(s): s \leq t_{1}\right] \\
\times \mathbb{E}\left[\frac{\alpha}{n} \int_{0}^{t_{1}}\left(m+\sum_{j=1}^{n-1} \frac{\lambda_{n}^{(m(n), n)}(s)+\lambda_{j}^{(n)}(s)}{\lambda_{n}^{(m(n), n)}(s)-\lambda_{j}^{(n)}(s)} \mathrm{d} s\right)\right\} \\
\left.\exp \left\{\alpha \int_{0}^{t_{2}} \sqrt{\frac{2 \lambda_{n}^{(m(n), n)}(s)}{n}} \mathrm{~d} W_{n}^{(n)}(s)\right\} \mid \sum_{m(n), n}(s): s \leq t_{1}\right] \geq
\end{gathered}
$$




$$
\begin{gathered}
\exp \left\{\frac{\alpha^{2}}{n} \int_{0}^{t_{1}} \lambda_{n}^{(m(n), n)}(s) \mathrm{d} s+\frac{\alpha}{n} \int_{0}^{t_{1}}\left(m+\sum_{j=1}^{n-1} \frac{\lambda_{n}^{(m(n), n)}(s)+\lambda_{j}^{(m(n), n)}(s)}{\lambda_{n}^{(m(n), n)}(s)-\lambda_{j}^{(m(n), n)}(s)} \mathrm{d} s\right)\right\} \\
\times \mathbb{E}\left[X_{\max }^{\alpha \sqrt{\frac{2}{n}}}\left(t_{2}\right) \mid \sum_{(m(n), n)}(s): s \leq t_{1}\right]= \\
\exp \left\{\frac{\alpha^{2}}{n} \int_{0}^{t_{1}} \lambda_{n}^{(m(n), n)}(s) \mathrm{d} s+\frac{\alpha}{n} \int_{0}^{t_{1}}\left(m+\sum_{j=1}^{n-1} \frac{\lambda_{n}^{(m(n), n)}(s)+\lambda_{j}^{(m(n), n)}(s)}{\lambda_{n}^{(m(n), n)}(s)-\lambda_{j}^{(m(n), n)}(s)} \mathrm{d} s\right)\right\} \\
\times X_{\max }^{\alpha \sqrt{\frac{2}{n}}}\left(t_{1}\right)=\exp \left(\alpha \lambda_{n}^{(m(n), n)}\left(t_{1}\right)\right),
\end{gathered}
$$

i.e., $\exp \left\{\alpha \lambda_{n}^{(m(n), n)}(t)\right\}_{t}$ is a submartingale.

The case of the process $t \rightarrow \exp \left(-\alpha \lambda_{1}^{(m(n), n)}(t)\right)$ follows similarly, by using (58).

Proof of Theorem 4.1. From (51) and Doob's inequality applied to the submartingale $\exp \left(\alpha \lambda_{n}^{(m(n), n)}(t)\right)$, we obtain

$$
\begin{gathered}
\mathbb{P}\left(\max _{0 \leq t \leq T} \lambda_{n}^{(m(n), n)}(t)>T\left(\varepsilon+\left(1+\sqrt{c_{n}}\right)^{2}\right)\right) \\
\leq \mathbb{P}\left(\max _{0 \leq t \leq T} \exp \left(\alpha \lambda_{n}^{(m(n), n)}(t)\right)>\exp \left\{\alpha T\left(\varepsilon+\left(1+\sqrt{c_{n}}\right)^{2}\right)\right\}\right) \\
\leq \exp \left\{-\alpha T\left(\varepsilon+\left(1+\sqrt{c_{n}}\right)^{2}\right)\right\} \mathbb{E}\left(\exp \left(\alpha \lambda_{n}^{(m(n), n)}(T)\right)\right) \\
\leq C n^{\frac{1}{2}} \exp \left(\alpha T\left(1+\sqrt{c_{n}}\right)^{2}+\frac{2 \alpha^{2} T^{2}\left(1+c_{n}\right)}{n}-\alpha T\left(\varepsilon+\left(1+\sqrt{c_{n}}\right)^{2}\right)\right) \\
=C n^{\frac{1}{2}} \exp \left(-\alpha T \varepsilon+\frac{2 \alpha^{2} T^{2}\left(1+c_{n}\right)}{n}\right)
\end{gathered}
$$

and the function (for $\varepsilon \in(0,1)$ )

$$
\alpha \longrightarrow \exp \left(-\alpha T \varepsilon+\frac{2 \alpha^{2} T^{2}\left(1+c_{n}\right)}{n}\right), \alpha>0,
$$

attains its minimum for $\alpha=\frac{n \varepsilon}{4 T\left(c_{n}+1\right)} \in\left(0, \frac{n}{4 T}\right]$, and replacing above, we get the inequality

$$
\mathbb{P}\left(\max _{0 \leq t \leq T} \lambda_{n}^{(m(n), n)}(t)>\varepsilon+\left(1+\sqrt{c_{n}}\right)^{2} T\right) \leq C n^{\frac{1}{2}} \exp \left(-\frac{n \varepsilon^{2}}{8\left(1+c_{n}\right)}\right) .
$$

Then, from (61), the convergence of the series $\sum_{n=1}^{\infty} n^{\frac{1}{2}} \exp \left(-\frac{n \varepsilon^{2}}{8\left(c_{n}+1\right)}\right)$ and Borel-Cantelli lemma, we obtain

$$
\limsup _{n \rightarrow \infty} \max _{0 \leq t \leq T} \lambda_{n}^{(m(n), n)}(t) \leq(1+\sqrt{c})^{2} T \text {, a.s.. }
$$


Next, from (36) we have that almost surely

$$
\lim _{n \rightarrow \infty} \max _{0 \leq t \leq T}\left|\frac{1}{n} \#\left\{1 \leq j \leq n: \lambda_{j}^{(m(n), n)}(t) \in\left[a_{1}, a_{2}\right]\right\}\right|=\max _{0 \leq t \leq T} \mu_{c}(t)\left(\left[a_{1}, a_{2}\right]\right),
$$

and then, also almost surely we have that

$$
\lim _{n \rightarrow \infty} \max _{0 \leq t \leq T} \#\left\{1 \leq j \leq n: \lambda_{j}^{(m(n), n)}(t) \in\left[(1+\sqrt{c})^{2} T-\varepsilon,(1+\sqrt{c})^{2} T\right]\right\} .
$$

Consequently

$$
\liminf _{n \longrightarrow \infty} \max _{0 \leq t \leq T} \lambda_{n}^{(m(n), n)}(t) \geq(1+\sqrt{c})^{2} T, \text { a.s.. }
$$

From (62), (63) we obtain (49).

Finally, (50) follows using similar arguments and the fact that the process $t \longrightarrow$ $\exp \left(-\alpha \lambda_{1}^{(m(n), n)}(t)\right)$ is a submartingale.

Acknowledgment. The authors thank the referee for a careful reading of the initial manuscript and for his/her very constructive and valuable suggestions.

\section{References}

[1] Bai Z.D.: Methodology in spectral analysis of large dimensional random matrices. Statistical Sinica 9 (1999), 611-677. MR1711663

[2] Bai Z.D., Yin Y.Q.: Limit of the smallest eigenvalue of a large dimensional sample covariance matrix. Ann. Probab. 21 (1993), 1275-1294. MR1235416

[3] Bai Z.D., Silverstein J.W. and Yin Y.Q.: A note on the largest eigenvalue of a large dimensional sample covariance matrix. J. Multivariate Anal. 26 (1988), 166-168. MR0963829

[4] Bru M.F.: Diffusions of perturbed principal component analysis. J. Multivariate Anal. 29 (1989), 127-136. MR0991060

[5] Cabanal Duvillard T., Guionnet A.: Large deviations upper bounds for the laws of matrixvalued processes and non-communicative entropies. Ann. Probab. 29 (2001), 1205-1261. MR1872742

[6] Capitaine M., Donati-Martin C.: Free Wishart processes. J. Theoret. Probab. 18 (2005), 413438. MR2137451

[7] Demni N.: The Laguerre process and generalized Hartman-Watson law. Bernoulli 13 (2007), 556-580. MR2331264

[8] Demni N.: Processus Stochastiques Matriciels, Systemes de Racines et Probabilités Non Commutatives. Thesis, Université Pierre et Marie Curie, 2007. 
[9] Donati-Martin C., Doumerc Y., Yor M.: Some properties of Wishart processes and a matrix extension of the Hartman-Watson laws. Publ. RIMS, Kyoto Univ. 40 (2004), 1385-1412. MR2105711

[10] Ethier S.N., Kurtz T.G.: Markov Processes: Characterization and Convergence. Wiley, 1986. New York. MR0838085

[11] Haagerup U., Thorbjørsen S.: Random matrices with complex Gaussian entries. Expo. Math. 21 (2003), 293-337. MR2022002

[12] Geman S.: A limit theorem for the norm of random matrices. Ann. Probab. 8 (1980), 252-261. MR0566592

[13] Graczyk P., Letac G. and Massam M.: The complex Wishart distribution and the symmetric group. Ann. Statist. 31 (2003), 287-309. MR1962508

[14] Graczyk P., Letac G. and Massam M.: The hyperoctahedral group, symmetric group representations and the moments of the real Wishart distribution. J. Theoret. Probab. 18 (2005), 1-42. MR2132270

[15] Graczyk P., Vostrikova L.: The moments of Wishart processes via Itô calculus. Theory Probab. Appl. 51 (2007), 609-625. MR2338064

[16] Guionnet A.: Random Matrices: Lectures on Macroscopic Asymptotics. École dÈté des Probabilités de Saint-Flour XXXVI 2006, (Lecture Notes in Mathematics), Springer, 2008.

[17] Hiai F., Petz D.: The Semicircle Law, Free Random Variables and Entropy. Mathematical Surveys and Monographs, Vol. 77. American Mathematical Society, Providence, R. I. 2000. MR1746976

[18] Jacod J., Shiryaev A.N.: Limit Theorems for Stochastic Processes. Springer-Verlag, Berlin, 1987. MR0959133

[19] Katori M., Tanemura, K.: Symmetry of matrix-valued stochastic processes and noncolliding diffusion particle systems. J. Math. Phys. 45 (2004), 3058-3085. MR2077500

[20] Konig W., O'Connell N.: Eigenvalues of the Laguerre process as non-coliding squared Bessel processes. Elec. Comm. Probab. 6 (2001), 107-114. MR1871699

[21] Lawi S.: Hermite and Laguerre polynomials and matrix-valued stochastic processes Bernoulli 13 (2008), 67-84. MR2386064

[22] Letac G., Massam H.: All invariants moments of the Wishart distribution. Scand. J. Statist. 31 (2004), 295-318. MR2066255

[23] Marchenko V.A., Pastur L.A.: The distribution of eigenvalues in certain sets of random matrices. Math. Sb. 72 (1967), 507-536. MR0208649

[24] Oravecz F., Petz P.: On the eigenvalue distribution of some symmetric random matrices. Acta Sci. Math. (Szeged) 63 (1997), 383-395. MR1480488 
[25] Pérez-Abreu V., Tudor C.: Functional limit theorems for trace processes in a Dyson Brownian motion. Comm. Stochast. Anal. 1 (2007), 415-428. MR2403859

[26] Silverstein J.W.: The smallest eigenvalue of a large dimensional Wishart matrix. Ann. Probab. 13 (1994), 1364-1368. MR0806232

[27] Voiculescu D., Dykema K.J. and Nica A.: Free Random Variables. CRM Monographs Series, Vol. 1, 1992. MR1217253

[28] Wachter K.W.: The strong limits of random matrix spectra for sample matrices of independent elements. Ann. Probab. 6 (1978), 1-18. MR0467894 